\title{
Connecting a Logical Framework to a First-Order Logic Prover ${ }^{\star}$
}

\author{
Andreas Abel, Thierry Coquand, and Ulf Norell \\ Department of Computing Science, Chalmers University of Technology \\ \{abel, coquand, ulfn\}@cs. chalmers.se
}

\begin{abstract}
We present one way of combining a logical framework and first-order logic. The logical framework is used as an interface to a first-order theorem prover. Its main purpose is to keep track of the structure of the proof and to deal with the high level steps, for instance, induction. The steps that involve purely propositional or simple first-order reasoning are left to a first-order resolution prover (the system Gandalf in our prototype). The correctness of this interaction is based on a general meta-theoretic result. One feature is the simplicity of our translation between the logical framework and first-order logic, which uses implicit typing. Implementation and case studies are described.
\end{abstract}

\section{Introduction}

We work towards human-readable and machine-verifiable proof documents for mathematics and computer science. As argued by de Bruijn [11], dependent type theory offers an ideal formal system for representing reasoning steps, such as introducing parameters or hypotheses, naming constants or lemmas, using a lemma or a hypothesis. Type theory provides explicit notations for these proof steps, with good logical properties. Using tools like Coq [5], Epigram [3], or Agda [9] these steps can be performed interactively. But low level reasoning steps, such as simple propositional reasoning, or equality reasoning, substituting equals for equals, are tedious if performed in a purely interactive way. Furthermore, propositional provers, and even first-order logic (FOL) provers are now very efficient. It is thus natural to create interfaces between logical frameworks and automatic propositional or first-order provers $[7,24,18]$. But, in order to arrive at proof documents which are still readable, only trivial proof steps should be handled by the automatic prover. Since different readers might have different notions of trivial, the automatic prover should not be a black box. With some effort by the human, the output of the prover should be understandable.

In this paper, we are exploring connections between a logical framework $M L F_{\text {Prop }}$ based on type theory and resolution-based theorem provers. One problem in such an interaction is that resolution proofs are hard to read and understand in general. Indeed, resolution proof systems work with formulæ in clause normal form, where clauses are (the universal closures of) disjunctions of literals, a literal being an atom or a negated

\footnotetext{
* Research supported by the coordination action TYPES (510996) and thematic network Applied Semantics II (IST-2001-38957) of the European Union and the project Cover of the Swedish Foundation of Strategic Research (SSF).
} 
atom. The system translates the negation of the statement to be proved to clause form, using skolemisation and disjunctive normal form. It then generates new clauses using resolution and paramodulation, trying to derive a contradiction. If successful, the system does pruning on the (typically high number of) generated clauses and outputs only the relevant ones. ${ }^{1}$

We lose the structure of the initial problem when doing skolemisation and clausification. Typically, a problem such as

$$
\forall x \cdot \exists y \cdot \forall z \cdot R(x, y) \Rightarrow R(x, z)
$$

is negated and translated into the two contradictory unit clauses

$$
\forall y . R(a, y), \quad \forall y \cdot \neg R(a, f(y)),
$$

but the connection between the statement (1) and the refutation of (2) is not so intuitive.

We do not solve this problem here, but we point out that, if we restrict ourselves to implicitely universally quantified propositional formulæ, in the following called open formulæ, this problem does not arise. Furthermore, when we restrict to this fragment, we can use the idea of implicit typing $[4,26]$. In this way, the translation from framework types to FOL formulæ is particularly simple. Technically, this is reflected by a general meta-theorem which ensures that we can lift a first-order resolution proof to a framework derivation. If we restrict the class of formulæ further to so-called geometrical open formulæ $[10,6]$, then the translation to clausal form is transparent. Indeed, any resolution proof for this fragment is intuitionistically valid and can be interpreted as it is in type theory. This meta-theorem is also the theoretical justification for our interface between $M_{\text {Lrop }}$ and a resolution-based proof system.

We have implemented a prototype version of a type system in Haskell, with a connection to the resolution prover Gandalf [25]. By restricting ourselves to open formulæ we sacrifice proof strength, but preliminary experiments show that the restriction is less severe than it may seem at first since the steps involving quantification are well handled at the framework level. Also, the proof traces produced by Gandalf are often readable (and surprisingly clever in some cases).

We think that we can represent Leslie Lamport proof style [17] rather faithfully in this system. The high level steps such as introduction of hypotheses, case analysis, induction steps are handled at the framework level, and only the trivial steps are sent to the FOL automatic prover.

One can think also of other plug-in extensions, e.g., rewriting systems and computer algebra systems. We have experimented with a QuickCheck [8] plug-in, that allows random testing of some propositions. In general, each plug-in extension of our logical framework should be justified in the same way as the one we present in this paper: we prove a conservativity result which ensures that the use of this plug-in can be, if desired, replaced by a direct proof in the framework. This way of combining various systems works in practice, as suggested by preliminary experiments, and it is theoretically wellfounded.

\footnotetext{
${ }^{1}$ If the search is not successful, it is quite hard to get any relevant information from the clauses that are generated. We have not yet analyzed the problem of getting useful feedback in this case.
} 
This paper is organized as follows. We first describe the logical framework MLF $F_{\text {Prop. }}$ We then present the translation from some LF types to FOL formulæ. The main technical result is then a theorem that shows that any resolution and paramodulation step, with one restriction, can be lifted to the framework level. Finally, we present some examples and extensions, and a discussion of related work.

\section{The Logical Framework MLF Prop}

This section presents an extension of Martin-Löf's logical framework [20] by propositions and local definitions.

Expressions (terms and types). We assume countable sets of variables Var and constants Const. Furthermore, we have a finite number of built-in constants to construct the primitives of our type language. A priori, we do not distinguish between terms and types. The syntactic entities of $M_{\text {L }} F_{\text {Prop }}$ are given by the following grammar.

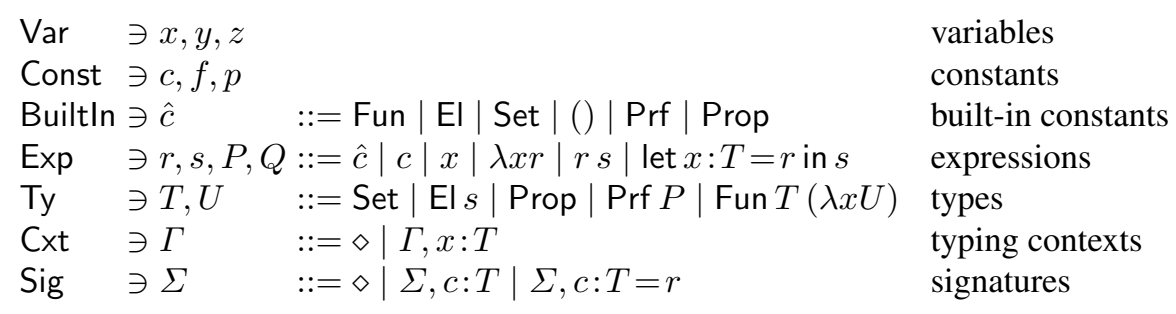

We identify terms and types up to $\alpha$-conversion and adopt the convention that in contexts $\Gamma$, all variables must be distinct; hence, the context extension $\Gamma, x: T$ presupposes $(x: U) \notin \Gamma$ for any $U$. Similarly, a constant $c$ may not be declared in a signature twice. We abbreviate a sequence of context entries $x_{1}: T, \ldots, x_{n}: T$ of the same type by $x_{1}, \ldots, x_{m}: T$. Multiple application $r s_{1} \ldots s_{n}$ is expressed as $r s$. (Capture-avoiding) substitution of $r$ for $x$ in $s$ is written as $s[r / x]$, or $s[r]$ if $x$ is clear from the context of discourse.

For dependent function types Fun $T(\lambda x U)$ we introduce the notation $(x: T) \rightarrow$ $U$. Curried functions spaces $\left(x_{1}: T_{1}\right) \rightarrow \ldots\left(x_{k}: T_{k}\right) \rightarrow U$ are shortened to $\left(x_{1}:\right.$ $\left.T_{1}, \ldots, x_{k}: T_{k}\right) \rightarrow U$, which explains the notation $(\Gamma) \rightarrow U$. Non-dependent functions $\left({ }_{-}: T\right) \rightarrow U$ are written $T \rightarrow U$. The inhabitants of Set are type codes; El maps type codes to types. E. g., $(a$ : Set $) \rightarrow \mathrm{El} a \rightarrow \mathrm{El} a$ is the type of the polymorphic identity $\lambda a \lambda x x$. Similarly Prop contains formal propositions $P$ and Prf $P$ proofs of $P$.

Types of the shape $(\Gamma) \rightarrow \operatorname{Prf} P$ are called proof types. A context $\Gamma:=x_{1}:$ $T_{1}, \ldots, x_{n}: T_{n}$ is a set context if and only if all $T_{i}$ are of the form $(\Delta) \rightarrow$ El $S$. In particular, if $P$ : Prop, then the proof type $(\Gamma) \rightarrow \operatorname{Prf} P$ corresponds to a universal first-order formula $\forall x_{1} \ldots \forall x_{n} P$ with quantifier-free kernel $P$. 
Judgements. The type theory MLF ${ }_{\text {Prop }}$ is presented via five judgements, which are all relative to a (user-defined) signature $\Sigma$.

$$
\begin{array}{ll}
\Gamma \vdash_{\Sigma} & \Gamma \text { is a well-formed context } \\
\Gamma \vdash_{\Sigma} T & T \text { is a well-formed type } \\
\Gamma \vdash_{\Sigma} r: T & r \text { has type } T \\
\Gamma \vdash_{\Sigma} T=T^{\prime} & T \text { and } T^{\prime} \text { are equal types } \\
\Gamma \vdash_{\Sigma} r=r^{\prime}: T & r \text { and } r^{\prime} \text { are equal terms of type } T
\end{array}
$$

All five judgements are defined simultaneously. Since the signature remains fixed in all judgements we will omit it. The typing rules are available in the extended version of this paper [2]. Judgmental type and term equality are generated from expansion of signature definitions as well as from $\beta-, \eta$-, and let-equality, the latter of which is given by (let $x: T=r$ in $s)=s[r / x]$. The rules for equality are similar to the ones of $\mathrm{MLF}_{\Sigma}$ [1], and type-checking of normal terms with local definitions is decidable.

Natural deduction. We assume a signature $\Sigma_{\mathrm{nd}}$ (see the extended version of this paper [2]) which assumes the infix logical connectives op ::= $\wedge, \vee, \Rightarrow$, plus the defined ones, $\neg$ and $\Leftrightarrow$. Furthermore, it contains a set PredSym of basic predicate symbols $p$ of type $(\Gamma) \rightarrow$ Prop where $\Gamma$ is a (possibly empty) set context. Currently we only assume truth $\top$, absurdity $\perp$, and typed equality Id, but user defined signatures can extend PredSym by their own symbols. For each logical constructs, there are appropriate proof rules, e. g., a constant impl : $(P, Q: \operatorname{Prop}) \rightarrow(\operatorname{Prf} P \rightarrow \operatorname{Prf} Q) \rightarrow \operatorname{Prf}(P \Rightarrow Q)$.

First-order logic assumes that every set is non-empty, and our use of a first-order prover is only sound under this assumption. Hence, we add a special constant $\epsilon:(D$ : Set $) \rightarrow$ El $D$ to $\Sigma_{\text {nd }}$ which enforces this fact. Notice that this implies that all set contexts are inhabited ${ }^{2}$.

Classical reasoning can be performed in the signature $\Sigma_{\text {class }}$, which we define as the extension of $\Sigma_{\text {nd }}$ by EM : $(P:$ Prop $) \rightarrow \operatorname{Prf}(P \vee \neg P)$, the law of the excluded middle.

The FOL rule. This article investigates conditions under which the addition of the following rule is conservative over $\mathrm{MLF}_{\text {Prop }}+\Sigma_{\text {nd }}$ and $\mathrm{MLF}_{\text {Prop }}+\Sigma_{\text {class }}$, respectively.

$$
\text { FOL } \frac{\Gamma \vdash T}{\Gamma \vdash(): T} \Gamma \vdash_{\mathrm{FOL}} T
$$

The side condition $\Gamma \vdash_{\text {FOL }} T$ expresses that $T$ is a proof type and that the first-order prover can deduce the truth of the corresponding first-order formula from the assumptions in $\Gamma$. It ensures that only tautologies have proofs in $\mathrm{MLF}_{\text {Prop }}$, but it is not considered part of the type checking. Meta-theoretical properties of $M L F_{\text {Prop }}$ like decidability of equality and type-checking hold independently of this side condition.

Conservativity fails if we have to compare proof objects during type-checking. This is because the rule FOL produces a single proof object for all (true) propositions, whereas upon removal of FOL the hole has to be filled with specific proof object. Hence two equal objects which each depend on a proof generated by FOL could

\footnotetext{
${ }^{2}$ Semantically, it may be fruitful to think of terms of type Set as inhabited Partial Equivalence Relations, while terms of type Prop are PERs with at most one inhabitant.
} 
become inequal after replacing FOL. To avoid this, it is sufficient to restrict function spaces $(x: T) \rightarrow U:$ if $T$ is a proof type, then also $U$.

In the remainder of the paper, we use $L F$ as a synonym for $M L F_{\text {Prop. }}$.

\section{Translation from $M L F_{\text {Prop }}$ to FOL}

We shall define a partial translation from some LF types to FOL propositions. We translate only types of the form

$$
\left(x_{1}: T_{1}, \ldots, x_{k}: T_{k}\right) \rightarrow \operatorname{Prf}\left(P\left(x_{1}, \ldots, x_{k}\right)\right),
$$

and these are translated to open formulæ $\left[P\left(x_{1}, \ldots, x_{k}\right)\right]$ of first-order logic. All the variables $x_{1}, \ldots, x_{k}$ are considered universally quantified. For instance,

$$
(x: \text { EI N }) \rightarrow \operatorname{Prf}(\text { Id N } x x \wedge \text { Id N } x(\text { add } 0 x))
$$

will be translated to $x=x \wedge x=\operatorname{add} 0 x$. If we have a theory of lattices, that is, we have added

$$
\begin{aligned}
& D \quad: \text { Set } \\
& \text { sup : El } D \rightarrow \text { El } D \rightarrow \text { El } D \\
& \leq \quad: \text { El } D \rightarrow \text { El } D \rightarrow \text { Prop }
\end{aligned}
$$

to the current signature, then $(x, y:$ El $D) \rightarrow \operatorname{Prf}(\sup x y \leq x \Leftrightarrow y \leq x)$ would be translated to sup $x y \leq y \Leftrightarrow y \leq x$.

The translation is done at a syntactical level, without using types. We will demonstrate that we can lift a resolution proof of a translated formula to a LF derivation in the signature $\Sigma_{\text {class }}$ (or in $\Sigma_{\text {nd }}$, in some cases).

\subsection{Formal Description of the Translation}

We translate normal expressions, which means that all definitions have been unfolded and all redexes reduced. Three classes of normal $\mathrm{MLF}_{\text {Prop }}$-expressions are introduced: (formal) first-order terms and (formal) first-order formula, which are quantifier free formulæ over atoms possibly containing free term variables, and translatable formulae, which are first-order formulæ prefixed by quantification over set elements.

$$
\begin{aligned}
& t, u \quad::=x \mid f \boldsymbol{t} \quad \text { first-order terms } \\
& A, B::=p \boldsymbol{t} \mid \operatorname{Id} S t_{1} t_{2} \quad \text { atoms } \\
& W \quad::=A \mid W \text { op } W^{\prime} \quad \text { first-order formulæ } \\
& \phi \quad::=(\Delta) \rightarrow \operatorname{Prf} W \quad \text { translatable formulæ }(\Delta \text { set context })
\end{aligned}
$$

Proper terms are those which are not just variables. For the conservativity result the following fact about proper terms will be important: In a well-typed proper term, the types of its variables are uniquely determined. For this reason, a formal first-order term $t$ may neither contain a binder ( $\lambda$ or let) nor a variable which is applied to something, for instance, $x u$. 
An example of a first-order formula is $W_{\text {ex }}:=\operatorname{ld} D x(f y) \Rightarrow($ Less $x(f y) \Rightarrow \perp)$, which is well-typed in the extension $D$ : Set, $f: \mathrm{El} D \rightarrow \mathrm{El} D$, Less : El $D \rightarrow$ El $D \rightarrow$ Prop of signature $\Sigma_{\text {nd }}$.

On the FOL side, we consider a language with equality $(=)$, one binary function symbol app and one constant for each constant introduced in the logical framework. Having an explicit "app" allows partial application of function symbols.

Let $\Delta=x_{1}: T_{1}, \ldots, x_{n}: T_{n}$ be a set context. A type of the form

$$
\phi:=(\Delta) \rightarrow \operatorname{Prf} W
$$

is translated into a universal formula $[\phi]=\forall x_{1} \ldots \forall x_{n}[W]$. The translation $[W]$ of first-order formulæ and the translation $\langle t\rangle$ of first-order terms depends on $\Delta$ and is defined recursively as follows:

$$
\begin{array}{rlrl}
{\left[W_{1} \text { op } W_{2}\right]} & :=\left[W_{1}\right] \text { op }\left[W_{2}\right] & & \text { logical connectives } \\
{\left[\operatorname{ld} S t_{1} t_{2}\right]} & :=\left\langle t_{1}\right\rangle=\left\langle t_{2}\right\rangle & & \text { equality } \\
{\left[p t_{1} \ldots t_{n}\right]} & :=p\left(\left\langle t_{1}\right\rangle, \ldots,\left\langle t_{n}\right\rangle\right) & & \text { predicates, including } \top, \perp \\
\left\langle x_{i}\right\rangle & :=x_{i} & & \text { variables in } \Delta \\
\langle x\rangle & :=c_{x} & & \text { variables not in } \Delta \\
\langle c\rangle & :=c & 0 \text {-ary functions } \\
\left\langle f t_{1} \ldots t_{n}\right\rangle:=f\left(\left\langle t_{1}\right\rangle, \ldots,\left\langle t_{n}\right\rangle\right) & & \text { n-ary functions }
\end{array}
$$

where we write $f\left(t_{1}, \ldots, t_{n}\right)$ for $\operatorname{app}\left(\ldots \operatorname{app}\left(\operatorname{app}\left(f, t_{1}\right), t_{2}\right), \ldots, t_{n}\right)$. Note that the translation is purely syntactical, and does not use type information. It is even homomorphic with two exceptions: (a) the typed equality of MLF $F_{\text {Prop }}$ is translated into the untyped equality of FOL, and (b) variables bound outside $\phi$ have to be translated as constants.

For instance, the formula $(y:$ El $D) \rightarrow W_{\text {ex }}$ is translated as $\forall y . c_{x}=f(y) \Rightarrow$ (Less $\left.\left(c_{x}, f(y)\right) \Rightarrow \perp\right)$. Examples of types that cannot be translated are

$$
(x: \text { Prop }) \rightarrow \operatorname{Prf} x, \quad \operatorname{Prf}(F(\lambda x x)), \quad(y: \text { El } D \rightarrow \text { El } D) \rightarrow \operatorname{Prf}(P(y x)) .
$$

We shall also use the class of geometrical formula, given by the following grammar:

$$
\begin{array}{ll}
G::=H|H \rightarrow G| G \wedge G & \text { geometrical formula } \\
H::=A|H \wedge H| H \vee H & \text { positive formula }
\end{array}
$$

The above example $W_{\text {ex }}$ is geometrical. As we will show, (classical) first-order proofs of geometrical formulæ can be mapped to intuitionistic proofs in the logical framework with $\Sigma_{\text {nd }}$.

\subsection{Resolution Calculus}

It will be convenient to use the following non-standard presentation of the resolution calculus [22]. A clause $C$ is an open first-order formula of the form

$$
A_{1} \wedge \cdots \wedge A_{n} \Rightarrow B_{1} \vee \cdots \vee B_{m}
$$


where we can have $n=0$ or $m=0$ and $A_{i}$ and $B_{j}$ are atomic formulæ. Following Gentzen [12], we write such a clause on the form

$$
A_{1}, \ldots, A_{n} \Rightarrow B_{1}, \ldots, B_{m}
$$

that is, $X \Rightarrow Y$, where $X$ and $Y$ are finite sets of atomic formulæ. An empty $X$ is interpreted as truth, an empty $Y$ as absurdity.

Resolution is forward reasoning. Figure 1 lists the rules for extending the current set of derived clauses: if all clauses mentioned in the premise of a rule are present, this rule can fire and the clause of the conclusion is added to the clause set.

$$
\begin{gathered}
\quad \operatorname{AX} \frac{\operatorname{sUB} \frac{X^{\prime} \supseteq X \quad X \Rightarrow Y \quad Y \subseteq Y^{\prime}}{A \Rightarrow A} \quad X^{\prime} \Rightarrow Y^{\prime}}{\operatorname{RES} \frac{X_{1} \Rightarrow Z_{1}, Y_{1} \quad X_{2}, Z_{2} \Rightarrow Y_{2}}{\left(X_{1}, X_{2} \Rightarrow Y_{1}, Y_{2}\right) \sigma} \sigma=\operatorname{mgu}\left(Z_{1}, Z_{2}\right)} \\
\operatorname{REFL} \frac{X_{1} \Rightarrow t=u, Y_{1} \quad X_{2}\left[t^{\prime}\right] \Rightarrow Y_{2}\left[t^{\prime}\right]}{\left(X_{1}, X_{2}[u] \Rightarrow Y_{1}, Y_{2}[u]\right) \sigma} \sigma=\operatorname{mgu}\left(t, t^{\prime}\right)
\end{gathered}
$$

Fig. 1. Resolution calculus.

In our formulation, all rules are intuitionistically valid ${ }^{3}$, and can be justified in $\mathrm{MLF}_{\text {Prop }}+\Sigma_{\text {nd }}$. It can be shown, classically, that these rules are complete in the following sense: if a clause is a semantical consequence of other clauses then it is possible to derive it using the resolution calculus. Hence, any proof in FOL can be performed with resolution ${ }^{4}$.

It can be pointed out that the SUB rule is only necessary at the very end-any resolution proof can be normalized to a proof that only uses SUB in the final step.

Let the restricted paramodulation rule denote the version of PARA where both $t$ and $t^{\prime}$ are proper terms (not variables).

\subsection{Proof of Correctness}

In this section, we show that every FOL proof of a translated formula $[\phi]$ can be lifted to a proof in $M_{\text {L }} F_{\text {prop }}+\Sigma_{\text {class }}$, provided the resolution proof confines to restricted paramodulation. This is not trivial because FOL is untyped and MLF $F_{\text {Prop }}$ is typed, and our translation forgets the types. The crucial insight is that every resolution step preserves well-typedness.

\footnotetext{
${ }^{3}$ In the standard formulation, the AX rule would read $\neg A \vee A$ - the excluded middle.

${ }^{4}$ To deal with existential quantification we also need skolemisation.
} 
Fix a signature $\Sigma$. A first-order term $t$ is well-typed iff there exists a context $\Delta$, giving types to the variables $x_{1}, \ldots, x_{n}$ of $t$, such that in the given signature, $\Delta \vdash t: T$ for some type $T$. For example, in the signature

$$
\begin{array}{ll}
D: \text { Set } & f: \text { El } D \rightarrow \text { El } D \\
F: \text { El } D \rightarrow \text { Prop } & g:(x: \text { El } D) \rightarrow \text { Prf }(F x)
\end{array}
$$

the proper first-order terms $f x, F y$, and $g z$ are well-typed, but $F x y$ is not. Notice that if a proper FOL term is well-typed, then there is only one way to assign types to its variables.

Lemma 1. If two proper first-order terms $t_{1}, t_{2}$ over disjoint variables are well-typed and unifiable, then the most general unifier $\mathrm{mgu}\left(t_{1}, t_{2}\right)$ is well-typed.

For instance, add $x 0$ and add (S $y) z$ are unifiable and well-typed and the most general unifier $\{x \mapsto S y, z \mapsto 0\}$ is well-typed. The lemma is proven in the extended version of this paper [2].

Using this lemma, we can lift any FOL resolution step to an LF resolution step. The same holds for any restricted paramodulation step, which justifies the translation of Id $S t u$ as $\langle t\rangle=\langle u\rangle$ in FOL, Indeed, in the paramodulation step between $X_{1} \Rightarrow$ $t=u, Y_{1}$ and $X_{2}\left[t^{\prime}\right] \Rightarrow Y_{2}\left[t^{\prime}\right]$ we unify $t$ and $t^{\prime}$ and for Lemma 1 to be applicable both $t$ and $t^{\prime}$ have to be proper terms. Similar arguments have been put forth by Beeson [4] and Wick and McCune [26].

A clausal type is a formula which translates to a clause.

Lemma 2. If two FOL clausal types $\left(\Gamma_{1}\right) \rightarrow \operatorname{Prf}\left(W_{1}\right)$ and $\left(\Gamma_{2}\right) \rightarrow \operatorname{Prf}\left(W_{2}\right)$ are derivable, and $C$ is a resolution of $\left[W_{1}\right]$ and $\left[W_{2}\right]$ then there exists a context $\Gamma$ and $a$ derivable $(\Gamma) \rightarrow \operatorname{Prf} W$ such that $C=[W]$. The same holds if $C$ is derived from $\left[W_{1}\right]$ and $\left[W_{2}\right]$ by restricted paramodulation. Furthermore in both cases, $\Gamma$ is a set context if both $\Gamma_{1}$ and $\Gamma_{2}$ are set contexts.

In the next theorems, $\phi, \phi_{1}, \ldots, \phi_{k}$ are translatable formulæ of the form $(\Gamma) \rightarrow$ Prf $W$ where $\Gamma$ is a set context.

The following theorem is a consequence of Lemma 2, since an open formula is (classically) equivalent to a conjunction of clauses.

Theorem 3. If we can derive $[\phi]$ from $\left[\phi_{1}\right], \ldots,\left[\phi_{k}\right]$ by resolution and restricted paramodulation then $\phi$ is derivable from $\phi_{1}, \ldots, \phi_{k}$ in any extension of the signature $\Sigma_{\text {class. }}$.

A resolution proof, as we have presented it, is intuitionistically valid. The only step which may not be intuitionistically valid is when we express the equivalence between an open formula and a conjunction of clauses. For instance the open formula $\neg P \vee Q$ is not intuitionistically equivalent to the clause $P \Rightarrow Q$ in general. This problem does not occur if we start with geometrical formulæ [6].

Theorem 4. If we can derive $[\phi]$ from $\left[\phi_{1}\right], \ldots,\left[\phi_{k}\right]$ by resolution and restricted paramodulation and $\phi, \phi_{1}, \ldots, \phi_{k}$ are geometric formula, then $\phi$ is derivable from $\phi_{1}, \ldots, \phi_{k}$ in any extension of the signature $\Sigma_{\mathrm{nd}}$. 
It is important for the theorem that all set contexts are inhabited: if $D:$ Set and $P$ : Prop (with $x$ not free in $P$ ), then both

$$
\phi_{1}=(x: \text { El } D) \rightarrow \operatorname{Prf} P \text { and } \phi_{2}=\operatorname{Prf} P
$$

are translated to the same FOL proposition $\left[\phi_{1}\right]=\left[\phi_{2}\right]=P$ but we can derive $\phi_{2}$ from $\phi_{1}$ in $\Sigma_{\mathrm{nd}}, D$ : Set, $P$ : Prop only because El $D$ is inhabited.

As noticed above, if we allow paramodulation from a variable, we could derive clauses that are not well-typed. For instance, in the signature

$$
N_{1}: \text { Set, } 0: \text { El } N_{1}, h:\left(x: \text { El } N_{1}\right) \rightarrow \operatorname{Prf}\left(\operatorname{Id} N_{1} x 0\right), A: \text { Set, } a: \text { El } A
$$

the type of $h$ becomes $x=0$ in FOL and from this we could derive, by paramodulation from the variable $x, a=0$ which is not well-typed. This problem is also discussed in $[4,26]$ and the solution is simply to forbid the FOL prover to use paramodulation from a variable ${ }^{5}$.

We can now state the conservativity theorem.

Theorem 5. If a type is inhabited in the system $\mathrm{MLF}_{\mathrm{Prop}}+\mathrm{FOL}+\Sigma_{\text {class }}$ then it is inhabited in $\mathrm{MLF}_{\text {Prop }}+\Sigma_{\text {class }}$.

Proof. By induction on the typing derivation, using Thm. 3 for FOL derivations.

\subsection{Simple Examples}

Figure 2 shows an extension of $\Sigma_{\text {nd }}$ by natural numbers, induction and an addition function defined by recursion on the second argument. Now consider the goal $(x$ : EI N) $\rightarrow$ Id N (add $0 x$ ) $x$. Using the induction schema and the propositional proof rules, we can give the proof term

$$
\operatorname{indN}(\lambda x \cdot \operatorname{ld} \mathrm{N}(\operatorname{add} 0 x) x)()(\lambda a \cdot \operatorname{impl}(\lambda i h()))
$$

in the logical framework, which contains these two FOL goals:

$$
\begin{aligned}
& \left.\vdash_{\text {FOL }} \text { Id N (add } 00\right) 0 \\
& \left.\left.a \text { : El N, ih: Id N (add } 0 a) a \vdash_{\mathrm{FOL}} \text { Id N (add } 0 \text { (S } a\right)\right)(\mathrm{S} a)
\end{aligned}
$$

Both goals can be handled by the FOL prover. The first goal becomes add $00=0$ and is proved from add $x 0=x$, the translation of axiom add 0 . The second goal becomes add $0(\mathrm{~S} a)=\mathrm{S} a$. This is a first-order consequence of the translated induction hypothesis add $0 a=a$ and add $x(\mathrm{~S} y)=\mathrm{S}(\operatorname{add} x y)$, the translation of axiom addS.

This example, though very simple, is a good illustration of the interaction between LF and FOL: the framework is used to handle the induction step and in the second goal, the introduction of the parameter $a$ and the induction hypothesis.

\footnotetext{
${ }^{5}$ This is possible in Otter. In Gandalf, this could be checked from the trace. Paramodulation from a variable is highly non-deterministic. For efficiency reasons, it was not present in some version of Gandalf, but it was added later for completeness. In the examples we have tried, this restriction is not a problem.
} 


$$
\begin{aligned}
& \mathrm{N} \text { : Set natural numbers } \\
& 0 \quad: \text { EIN zero } \\
& \mathrm{S} \quad: \mathrm{EIN} \rightarrow \mathrm{EIN} \text { successor } \\
& \text { indN }:(P: \text { El N } \rightarrow \text { Prop }) \rightarrow P 0 \\
& \rightarrow((x: \mathrm{EIN}) \rightarrow P x \Rightarrow P(\mathrm{~S} x)) \\
& \rightarrow(n: \mathrm{EI} \mathrm{N}) \rightarrow P n \quad \text { induction } \\
& \text { add }: \mathrm{EIN} \rightarrow \mathrm{EIN} \rightarrow \mathrm{EIN} \quad \text { addition } \\
& \text { add0 }:(x \quad: \text { EI N }) \rightarrow \text { Id N (add } x 0) x \quad \text { axiom } 1 \text { of add } \\
& \text { addS }:(x, y: \text { EI } \mathrm{N}) \rightarrow \text { Id } \mathrm{N}(\text { add } x(\mathrm{~S} y))(\mathrm{S}(\text { add } x y)) \quad \text { axiom } 2 \text { of add }
\end{aligned}
$$

Fig. 2. A Signature of Natural Numbers and Addition.

Here is another simple example which illustrates that we can call the FOL prover even in a context involving non first-order operations. This example comes from a correctness proof of Warshall's algorithm. Let $D$ : Set.

$$
\begin{aligned}
& F: \text { El } D \rightarrow(\text { El } D \rightarrow \text { El } D \rightarrow \text { Prop }) \rightarrow \text { El } D \rightarrow \text { El } D \rightarrow \text { Prop } \\
& F a R x y=R x y \vee(R x a \wedge R a y) \\
& \text { swap }:(a, b, x, y: \text { El } D) \rightarrow \operatorname{Prf}(F a(F b R) x y \Leftrightarrow F b(F a R) x y)
\end{aligned}
$$

The operation $F$ is a higher-order operation. However, in the context $R: \operatorname{El} D \rightarrow$ EI $D \rightarrow$ Prop, the goal swap can be handled by the FOL prover. The normal form of $F a(F b R) x y \Leftrightarrow F b(F a R) x y$, where all defined constants (here only $F$ ) have been unfolded, is a translatable formula.

\section{Implementation}

To try out the ideas described in this paper we have implemented a prototype type checker in Haskell. In addition to the logical framework, the type checker supports implicit arguments and the extensions described in Section 6: sigma types, datatypes and definitions by pattern matching.

\subsection{Implicit Arguments}

A problem with LF as presented here is its rather heavy notation. For instance, to state that function composition is associative one would give the signature in Figure 3. This is very close to being completely illegible due to the fact that we have to be explicit about the type arguments to the composition function. To solve the problem, we have implemented a mechanism for implicit arguments which allows the omission of arguments that can be inferred automatically. Using this mechanism the associativity example can be written as follows: 


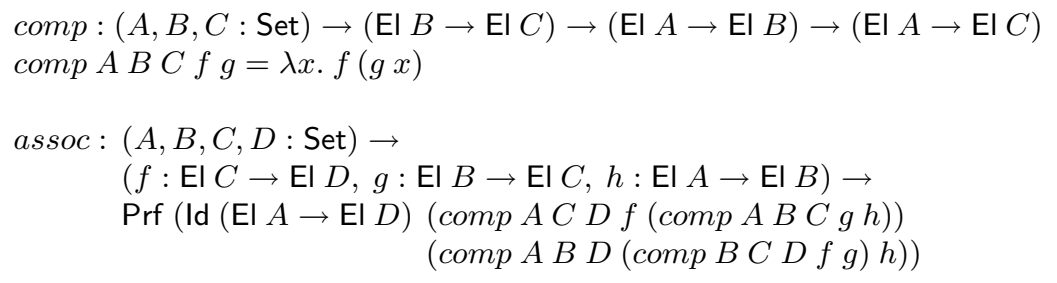

Fig. 3. Associativity without Implicit Arguments.

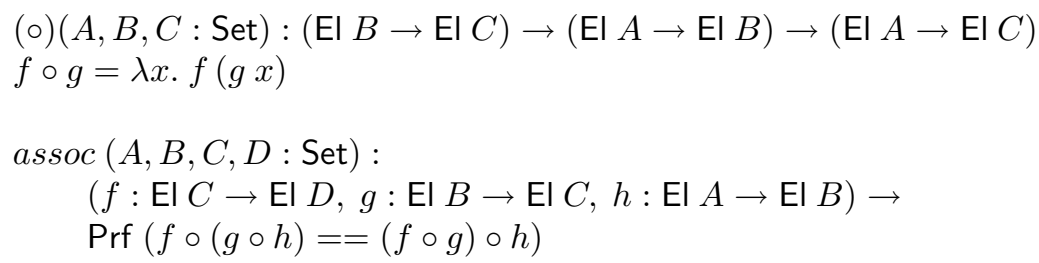

In general, we write $x(\Delta): T$ to say that $x$ has type $(\Delta) \rightarrow T$ with $(\Delta)$ implicit. The scope of the variables in $\Delta$ extends to the definition of $x$ (if there is one). For every use of $x$ we require that the instantiation of $(\Delta)$ can be inferred using pattern unification [19]. Note that when we have implicit arguments we can replace Id with an infix operator $(==)(D$ : Set $):$ El $D \rightarrow$ El $D \rightarrow$ Prop

We conjecture that the conservativity result can be extended to allow the omission of implicit arguments when translating to first-order logic if they can be inferred from the resulting first-order term. In this case we preserve the property that for a well-typed FOL term there exists a unique typing, which is an important lemma in the conservativity theorem. The kind of implicit arguments we work with can most often be inferred in this way. It is doubtful, however, that it would work for other kinds of implicit arguments such as implicit dictionaries used for overloading.

Omitting the implicit arguments, the formula $f \circ(g \circ h)=(f \circ g) \circ h$ in the context $A, B, C, D:$ Set, $f:$ El $C \rightarrow$ El $D, g:$ El $B \rightarrow$ El $C, h:$ El $A \rightarrow$ El $B$ is translated to

$$
f \circ(g \circ h)=(f \circ g) \circ h
$$

With this translation, the first-order proofs are human readable and, in many cases, correspond closely to a pen and paper proof.

\subsection{The Plug-in Mechanism}

The type checker is equipped with a general plug-in interface that makes it easy to experiment with connections to external tools. A plug-in should implement two functions: 
a type checking function which can be called on particular goals in the program, and a finalization function which is called after type checking.

To control where the type checking function of a plug-in is invoked we introduce a new form of expressions:

$$
\operatorname{Exp}::=\ldots \mid \text { name }-\operatorname{plugin}\left(s_{1}, \ldots, s_{n}\right) \quad \text { invoking a plug-in }
$$

where name is the name of a plug-in. It is possible to pass arguments $\left(s_{1}, \ldots, s_{n}\right)$ to the plug-in. These arguments can be arbitrary expressions which are ignored by the type checker. Hence it is possible to pass ill-typed terms as arguments to a plug-in; it is the responsibility of the plug-in to interpret the arguments. Most plug-ins, of course, expect well-typed arguments and in this case, the plug-in has to invoke the type checker explicitly on its arguments.

\subsection{The FOL Plug-in}

The connection between LF and FOL has been implemented as a plug-in using the mechanism described above. With this implementation we replace the built-in constant () by a call to the plug-in. The idea is that the plug-in should be responsible for checking the side condition $\Gamma \vdash_{\mathrm{FOL}} P$ in the FOL rule.

An important observation is that decidability of type checking and equality do not depend on the validity of the propositions being checked by the FOL plug-in-nothing will break if the type checker is led to believe that there is an $s: \operatorname{Prf} \perp$. This allows us to delay all first-order reasoning until after type checking. The rationale for doing this is that type checking is cheap and first-order proving is expensive.

Another observation is that it is not feasible to pass the entire context to the prover. Typically, the context contains lots of things that are not needed for the proof, but would rather overwhelm the prover. To solve this problem, we require that any axioms or lemmas needed to prove a particular goal are passed as arguments to the plug-in. This might seem a severe requirement, but bear in mind that the plug-in is intended for simple goals where you already have an idea of the proof.

More formally, the typing rule for calls to the FOL plug-in is

$$
\frac{\Gamma \vdash \phi \quad \Gamma \vdash s_{1}: \phi_{1} \ldots \Gamma \vdash s_{n}: \phi_{n}}{\Gamma \vdash \text { fol-plugin }\left(s_{1}, \ldots, s_{n}\right): \phi} \phi_{1}, \ldots, \phi_{n} \vdash_{\mathrm{FOL}} \phi .
$$

When faced with a call to a plug-in the type checker calls the type checking function of the plug-in. In this case, the type checking function of the FOL plug-in will verify that the goal is a translatable formula and that the arguments are well-typed proofs of translatable formulæ. If this is the case it will report success to the type checker and store away the side condition in its internal state. After type checking the finalization function of the FOL plug-in is called. For each constraint $\phi_{1}, \ldots, \phi_{n} \vdash_{\mathrm{FOL}} \phi$, this function verifies that $[\phi]$ is derivable from $\left[\phi_{1}\right], \ldots,\left[\phi_{n}\right]$ in the resolution calculus by translating the formulæ to clause normal form and feeding them to an external firstorder prover (Gandalf, at the moment). If the prover does not manage to find a proof within the given time limit, the plug-in reports an error. 


\section{Examples}

The code in this section has been type checked successfully by our prototype type checker. In fact, the typeset version is automatically generated from the actual code. The type checker can infer which types are Sets and which are Props, so we omit El and Prf in the types.

Natural numbers can be added to the framework by three new constants Nat, zero, succ plus an axiom for mathematical induction.

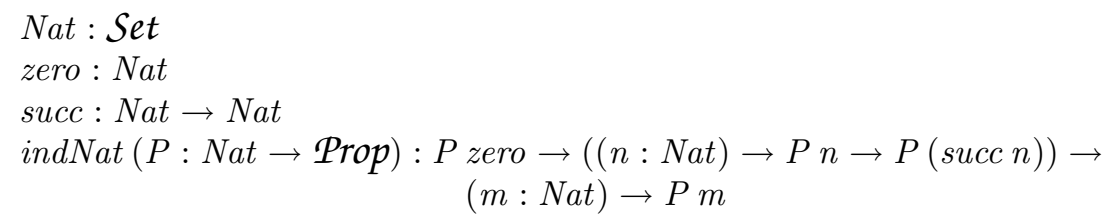

Now we fix a set $A$ and consider relations over $A$. We want to prove that the transitive closure of a symmetric relation is symmetric as well. We define the notion of symmetry and introduce a symbol for relation composition. We could define $R \circ R^{\prime}=\lambda x \lambda z \exists z \cdot x R y \wedge y R^{\prime} z$, but here we only assume that a symmetric relation composed with itself is also symmetric.

$$
\begin{aligned}
& A: \operatorname{Set} \\
& \text { sym }:(A \rightarrow A \rightarrow \text { Prop }) \rightarrow \text { Prop } \\
& \text { sym } R \\
& =( \\
& x \\
& \text { y } \\
& \text { A } \\
& ) \rightarrow \\
& R x y \\
& \Longrightarrow R y x \\
& (\circ):(A \rightarrow A \rightarrow \text { Prop }) \rightarrow(A \rightarrow A \rightarrow \text { Prop }) \rightarrow(A \rightarrow A \rightarrow \text { Prop })
\end{aligned}
$$


$\operatorname{axSymO}:(R: A \rightarrow A \rightarrow$ Prop $) \rightarrow \operatorname{sym} R \rightarrow \operatorname{sym}(R \circ R)$

We define a monotone chain of approximations $R^{(n)}$ (in the source: $R^{\wedge} n$ ) of the transitive closure, such that two elements will be related in the transitive closure if they are related in some approximation. The main lemma states that all approximations are symmetric, if $R$ is symmetric.

$$
\begin{aligned}
& \left({ }^{\wedge}\right):(A \rightarrow A \rightarrow \text { Prop }) \rightarrow N a t \rightarrow(A \rightarrow A \rightarrow \text { Prop }) \\
& \text { axTc }:(R: A \rightarrow A \rightarrow \text { Prop }) \rightarrow(x, y: A) \rightarrow(n: N a t) \rightarrow \\
& \quad\left(\left(R^{\wedge} \text { succ } n\right) x y \Leftrightarrow\left(R^{\wedge} n\right) x y \vee\left(\left(R^{\wedge} n\right) \circ\left(R^{\wedge} n\right)\right) x y\right) \\
& \wedge\left(\left(R^{\wedge} \text { zero } x y \Leftrightarrow R x y\right)\right. \\
& \text { main }:(R: A \rightarrow A \rightarrow \text { Prop }) \rightarrow \operatorname{sym} R \rightarrow(n: N a t) \rightarrow \operatorname{sym}\left(R^{\wedge} n\right) \\
& \text { main } R h=\text { indNat } \\
& \quad \text { fol-plugin }(h, \text { axTc } R) \\
& \quad\left(\lambda n i h \rightarrow \text { fol-plugin }\left(h, \text { axSymO }\left(R^{\wedge} n\right) i h, \text { axTc } R, i h\right)\right)
\end{aligned}
$$

Induction is performed at the framework level, base and step case are filled by Gandalf. Pretty printed, Gandalf produces the following proof of the step case:

(1) $\quad \forall x y \cdot\left(R^{(n)} \circ R^{(n)}\right) x y \Longrightarrow\left(R^{(n)} \circ R^{(n)}\right) y x$

(2) $\forall m x y \cdot R^{(\text {succm })} x y \Longrightarrow\left(R^{(m)} \circ R^{(m)}\right) x y \vee R^{(m)} x y$

(3) $\forall m x y \cdot\left(R^{(m)} \circ R^{(m)}\right) x y \Longrightarrow R^{(\text {succ } m)} x y$

(4) $\forall m x y \cdot R^{(m)} x y \Longrightarrow R^{(\text {succ } m)} x y$

(5) $\quad \forall x y \cdot R^{(n)} x y \Longrightarrow R^{(n)} y x$

(6) $\quad R^{(\operatorname{succ} n)} a b$

(7) $\quad R^{(\text {succ } n)} b a \Longrightarrow \perp$

(8) $\quad\left(R^{(n)} \circ R^{(n)}\right) a b \vee R^{(n)} a b$

(9) $\quad\left(R^{(n)} \circ R^{(n)}\right) b a \vee R^{(n)} a b$

(10) $\quad R^{(n)} a b$

(11) $\quad R^{(n)} b a$

$\perp$

The transitive closure is now defined as TC Rxy $y=\exists n \cdot R^{(n)} x y$. To formalize this, we add existential quantification and its proof rules. The final theorem demostrates how existential quantification can be handled in the framework.

$$
\begin{aligned}
& \text { Exists }(X: \text { Set }):(X \rightarrow \text { Prop }) \rightarrow \text { Prop } \\
& \text { exists }(X: \text { Set })(P: X \rightarrow \text { Prop }):(x: X) \rightarrow P x \rightarrow \text { Exists } P \\
& \text { existsE }(X: \text { Set })(P: X \rightarrow \text { Prop })(C: \text { Prop }): \\
& \quad \text { Exists } P \rightarrow((x: X) \rightarrow P x \rightarrow C) \rightarrow C \\
& \text { TC: }(A \rightarrow A \rightarrow \text { Prop } \rightarrow A \rightarrow A \rightarrow \text { Prop } \\
& T C R x y=\text { Exists }\left(\lambda n \rightarrow\left(R^{\wedge} n\right) x y\right)
\end{aligned}
$$




$$
\begin{aligned}
& \text { thm }:(R: A \rightarrow A \rightarrow \text { Prop }) \rightarrow \operatorname{sym} R \rightarrow \operatorname{sym}(\text { TC } R) \\
& \text { thm Rhxy=impI }(\lambda p \rightarrow \\
& \text { existsE } p(\lambda n q \rightarrow \text { existsI } n \text { fol-plugin }(q, \text { main } R h n)))
\end{aligned}
$$

See the extended version [2] for an example involving algebra and induction.

\section{Related Work}

Smith and Tammet [24] also combine Martin-Löf type theory and first-order logic, which was the original motivation for creating the system Gandalf. The main difference to their work is that we use implicit typing and restrict to quantifier-free formulæ. An advantage is that we have a simple translation, and hence get a quite direct connection to resolution theorem provers. Hence, we can hope, and this has been tested positively in several examples, that the proof traces we get from the prover are readable as such and therefore can been used as a proof certificate or as feedback for the user. For instance, the user can formulate new lemmas suggested by this proof trace. We think that this aspect of readability is more important than creating an explicit proof term in type theory (which would actually be less readable). It should be stressed that our conservativity result contains, since it is constructive, an algorithm that can transform the resolution proof to a proof in type theory, if this is needed.

Huang et. al. [13] present the design of $\Omega-\mathrm{MKRP}^{6}$, a tool for the working mathematician based on higher-order classical logic, with a facility of proof planning, access to a mathematical database of theorems and proof tactics (called methods), and a connection to first-order automated provers. Their article is a well-written motivation for the integration of human and machine reasoning, where they envision a similar division of labor as we have implemented. We have, however, not addressed the problem of mathematical knowledge management and proof tactics.

Wick and McCune [26] list three options for connecting type systems and FOL: include type literals, put type functions around terms, or use implicit typing. We rediscovered the technique of implicit typing and found out later that it is present already in the work of Beeson [4]. Our work shows that this can also be used with dependent types, which is not obvious a priori. Our formulation of the correctness properties, as a conservativity statement, requires some care (with the role of the sort Prop), and is an original contribution.

Bezem, Hendriks, and de Nivelle [7] describe how to transform a resolution proof to a proof term for any first-order formula. However, the resulting proof terms are hard to read for a human because of the use of skolemisation and reduction to clausal forms. Furthermore, they restrict to a fixed first-order domain.

Hurd's work on a Gandalf-tactic for HOL [14] is along the same lines. He translates untyped first-order HOL goals to clause form, sends them to Gandalf and constructs an LCF proof from the Gandalf output. In later work $[15,16]$ he handles types by having two translations: the untyped translation, and a translation with explicit types. The typed translation is only used when the untyped translation results in an ill-typed proof.

\footnotetext{
${ }^{6}$ Markgraf Karl Refutation Procedure.
} 
JProver [23] is a connection-based intuitionistic theorem prover which produces proof objects. It has been integrated into NuPrl and Coq. The translation from type theory to first-order logic involves some heuristics when to include or discard type information. Unfortunately, the description [23] does not contain formal systems or correctness arguments, but focuses on the connection technology.

Jia Meng and Paulson [18] have carried out substantial experiments on how to integrate the resolution theorem prover Vampire into the interactive proof tool Isabelle. Their translation from higher-order logic (HOL) to first-order logic keeps type information, since HOL supports overloading via axiomatic type classes and discarding type information for overloaded symbols would lead to unsound reasoning. They claim to cut down the search space via type information, but this is also connected to overloading. The aim of their work is different to ours: while they use first-order provers to do as much automatic proofs and proof search as possible, we employ automation only to liberate the user from seemingly trivial proof steps.

In Coq, NuPrl, and Isabelle, the user constructs a proof via tactics. We provide type theory as a proof language in which the user writes down a proof skeleton, consisting of lemmas, scoped hypotheses, invokation of induction, and major proof steps. The firstorder prover is invoked to solve (easy) subgoals. This way, we hope to obtain humanreadable proof documents (see our examples).

\section{Conclusion and Future Work}

We have described the implementation of a logical framework with proof-irrelevant propositions and its connection to the first-order prover Gandalf. Soundness and conservativity of the connection have been established by general theorems.

It is natural to extend LF by sigma types, in order to represent, for instance, mathematical structures. The extension of the translation to FOL is straightforward, we simply add a new binary function symbols for representing pairs. A more substantial extension is the addition of data type and functions defined by case [21]. In this extension, it is possible to represent each connective as a parameterized data type. Each introduction rule is represented by a constructor, and the elimination rules are represented by functions defined by cases. This gives a computational justification of each of the axioms of the signature $\Sigma_{n a t}$. The extension of the translation to FOL is also straightforward: each defined equations for functions becomes a FOL equality. One needs also to express that each constructor is one-to-one and that terms with distinct constructors are distinct.

We plan to the extend the conservativity theorem to implicit arguments as presented in Section 3.1. We also think that we can extend our class of translatable formulæ, for instance, to include some cases of existential quantification.

One could think of adding more plug-ins, with the same principle that they are justified by a general meta-theorem. For instance, one could add a plug-in to a model checker, or a plug-in to a system with a decision procedure for Presburger arithmetic.

Acknowledgments. We thank the members of the Cover project, especially Koen Claessen for discussions on implicit typing and the clausification tool Santa for a uniform connection to FOL provers, and Grégoire Hamon for programming the clausifier of the FOL plug-in in a previous version. 


\section{References}

1. Andreas Abel and Thierry Coquand. Untyped algorithmic equality for Martin-Löf's logical framework with surjective pairs. In Paweł Urzyczyn, editor, TLCA'05, volume 3461 of LNCS, pages 23-38. Springer, April 2005.

2. Andreas Abel, Thierry Coquand, and Ulf Norell. Connecting a logical framework to a first-order logic prover (extended version). Technical report, Department of Computing Science, Chalmers University of Technology, Gothenburg, Sweden, 2005. Available under http://www.cs.chalmers.se/ ulfn/papers/fol.html.

3. Thorsten Altenkirch, Conor McBride, and James McKinna. Why dependent types matter. Manuscript, available online, April 2005.

4. Michael Beeson. Otter- $\lambda$ home page, 2005. URL: http://mh215a.cs.sjsu.edu/.

5. Yves Bertot and Pierre Castéran. Interactive Theorem Proving and Program Development. Coq'Art: The Calculus of Inductive Constructions. Texts in Theoretical Computer Science. An EATCS Series. Springer, 2004.

6. Marc Bezem and Thierry Coquand. Newman's lemma - a case study in proof automation and geometric logic. Bulletin of the EATCS, 79:86-100, 2003. Logic in Computer Science Column.

7. Marc Bezem, Dimitri Hendriks, and Hans de Nivelle. Automated proof construction in type theory using resolution. JAR, 29(3-4):253-275, 2002. Special Issue Mechanizing and Automating Mathematics: In honour of N.G. de Bruijn.

8. Koen Claessen and John Hughes. QuickCheck: a lightweight tool for random testing of Haskell programs. ACM SIGPLAN Notices, 35(9):268-279, 2000.

9. Catarina Coquand and Thierry Coquand. Structured type theory. In Workshop on Logical Frameworks and Meta-languages (LFM'99), Paris, France, September 1999.

10. Michel Coste, Henri Lombardi, and Marie-Françoise Roy. Dynamical methods in algebra: Effective Nullstellensätze. APAL, 111(3):203-256, 2001.

11. Niklas G. de Bruijn. A survey of the project Automath. In J. P. Seldin and J. R. Hindley, editors, To H. B. Curry: Essays in combinatory logic, lambda calculus and formalism, pages 579-606, London-New York, 1980. Academic Press.

12. Gerhard Gentzen. Untersuchungen über das logische Schließen. Mathematische Zeitschrift, 39:176-210, 405-431, 1935.

13. Xiaorong Huang, Manfred Kerber, Michael Kohlhase, Erica Melis, Dan Nesmith, Jörn Richts, and Jörg H. Siekmann. Omega-MKRP: A proof development environment. In Alan Bundy, editor, CADE'94, volume 814 of $L N C S$, pages 788-792. Springer, 1994.

14. Joe Hurd. Integrating Gandalf and HOL. In Yves Bertot, Gilles Dowek, André Hirschowitz, Christine Paulin, and Laurent Théry, editors, TPHOLS'99, volume 1690 of LNCS, pages 311-321. Springer, September 1999.

15. Joe Hurd. An LCF-style interface between HOL and first-order logic. In Andrei Voronkov, editor, $C A D E^{\prime} 02$, volume 2392 of $L N A I$, pages 134-138. Springer, 2002.

16. Joe Hurd. First-order proof tactics in higher-order logic theorem provers. In Myla Archer, Ben Di Vito, and César Muñoz, editors, STRATA'03, number CP-2003-212448 in NASA Technical Reports, pages 56-68, September 2003.

17. Leslie Lamport. How to write a proof. In Global Analysis in Modern Mathematics, pages 311-321. Publish or Perish, Houston, Texas, U.S.A., February 1993. Also appeared as SRC Research Report 94.

18. Jia Meng and Lawrence C. Paulson. Experiments on supporting interactive proof using resolution. In David A. Basin and Michaël Rusinowitch, editors, IJCAR'04, volume 3097 of LNCS, pages 372-384. Springer, 2004.

19. Dale Miller. Unification under a mixed prefix. J. Symb. Comput., 14(4):321-358, 1992. 
20. Bengt Nordström, Kent Petersson, and Jan Smith. Martin-Löf's type theory. In Handbook of Logic in Computer Science, volume 5. OUP, October 2000.

21. Bengt Nordström, Kent Petersson, and Jan M. Smith. Programming in Martin Löf's Type Theory: An Introduction. Clarendon Press, Oxford, 1990.

22. John Alan Robinson. A machine-oriented logic based on the resolution principle. JACM, 12(1):23-41, January 1965.

23. Stephan Schmitt, Lori Lorigo, Christoph Kreitz, and Aleksey Nogin. JProver: Integrating connection-based theorem proving into interactive proof assistants. In R. Gore, A. Leitsch, and T. Nipkow, editors, IJCAR'01, volume 2083 of LNAI, pages 421-426. Springer, 2001.

24. Jan M. Smith and Tanel Tammet. Optimized encodings of fragments of type theory in firstorder logic. In Stefano Berardi and Mario Coppo, editors, TYPES'95, volume 1158 of LNCS, pages 265-287. Springer, 1995.

25. Tanel Tammet. Gandalf. JAR, 18(2):199-204, 1997.

26. Cynthia A. Wick and William McCune. Automated reasoning about elementary point-set topology. JAR, 5(2):239-255, 1989. 